\title{
Late life depression: nursing actions that can help
}

\author{
Sherri Melrose PhD, RN
}

Faculty of Health Disciplines, Centre for Nursing and Health Studies, Athabasca University, Athabasca, Alberta

\section{Correspondence}

Sherri Melrose PhD, RN, Faculty of Health Disciplines, Athabasca University, 1 University Drive, Athabasca, AB T9S 3A3, Canada.

Email: sherrim@athabascau.ca

\begin{abstract}
Purpose: This article explains the symptoms of late life depression (LLD) and discusses evidence-informed actions that nurses can implement to provide older adults with the help they need.

Conclusions: Recognizing and addressing depression in older adults can enhance quality of life.

Practice Implications: People with LLD may not appear sad or express feelings of depression. Instead, they demonstrate loss of interest, frailty, cognitive impairment, suicidal ideation, unexplained somatic complaints, and loneliness. Documenting symptoms, screening, and assessing suicidal ideation are essential. Positive outcomes are associated with antidepressant medications, cognitive behavioral therapy, electroconvulsive therapy, neuromodulation therapies, and exercise.
\end{abstract}

\section{KEYWORDS}

late life depression, LLD, nursing care for older adults

\section{1 | INTRODUCTION}

Late life depression (LLD) is the occurrence of a major depressive disorder in adults aged 60 years or older. ${ }^{1}$ When symptoms that do not meet major depressive disorder criteria are also considered, subsyndromal depressive disorders and clinically significant symptoms of depression in later life are estimated to affect between $0.9 \%$ and $49.0 \%$ of adults aged 65 years or older. ${ }^{2-4}$ As many as $17 \%$ of adults over $75 ; 25 \%$ of adults over 85 ; and $49 \%$ of adults over 90 are affected by at least some symptoms of depression. ${ }^{2,5}$

LLD is associated with cognitive impairment, increased morbidity and mortality from co-occurring medical illness, increased death by suicide as well as increased health care utilization and cost. 1,3,6 Depression in older adults is often undetected or inadequately treated..$^{7-13}$ Existing literature has focused on providing physicians with information related to diagnosing and treating LLD. However, nurses in a variety of practice areas also encounter many older adults who are struggling to cope with symptoms of depression. Psychiatric and mental health nurses are well positioned to provide these nurse peers with a deeper understanding of the complexities of the condition. When nurses recognize LLD and implement evidenceinformed care, they are better able to support the older adults in their care toward an improved quality of life. This article presents an explanation of LLD and suggests nursing actions that can help.

\section{IOWARD AN EXPLANATION OF LLD}

People experience depression later in their lives in different ways and for a variety of different reasons. Explanations of LLD are seldom straightforward. LLD is generally explained as depression that occurs after the age of 60 years, although onset and definition of cutoff may vary. ${ }^{14}$ Presentations of LLD can include reoccurrence of a previous depression, termed early onset depression; a mood disorder secondary to a general medical condition; a depressed mood secondary to a substance or medication use (including polypharmacy); or as a new-onset depression, later in life, termed late-onset depression (LOD). ${ }^{5}$ As many as half of the cases of LLD are late onset and are occurring among people who have not previously experienced clinically significant symptoms of depression. ${ }^{15}$ In this article, the term LLD will refer to LOD. Unless otherwise specified, the information presented can be considered relevant to the care of older people experiencing any form of depression.

Both the diagnosis of major depressive disorder (MDD) and the presence of subsyndromal or clinically significant depressive 
symptoms are associated with LLD. ${ }^{15}$ According to the Diagnostic and Statistical Manual of Mental Disorders, 5th edition (DSM-5), ${ }^{16}$ to receive a diagnosis of $M D D$, an individual must exhibit symptoms of depressed mood and/or loss of interest plus four or more associated symptoms, including changes in appetite, sleep disturbance, psychomotor agitation or retardation, fatigue, inappropriate guilt or feelings of worthlessness, poor concentration or indecisiveness, and recurrent thoughts of death or suicidal ideation. Symptoms must be present nearly every day for at least two weeks and cause clinically significant distress or functional impairment. ${ }^{16}$

However, in older adults, the two core symptoms of MDD, depressed mood (sadness or dysphoria) and loss of interest in activities once found pleasurable (anhedonia) can differ from those seen in younger people. ${ }^{17}$ The affective symptom of a "depressed mood" can actually be absent in some older adults who are suffering from depression. Rather, a lack of sadness or dysphoria, known as depression without sadness, where mood does not appear sad is common. ${ }^{18-20}$ Similarly, rather than clear indications of sadness, symptoms like lack of vigor, apathy, and withdrawal, known as "depletion syndrome" are apparent. ${ }^{19}$

In contrast, the second MDD core symptom of "loss of interest" is usually pronounced in LLD. ${ }^{17}$ Clinicians, family members, and older adults themselves may inadvertently view anhedonia more as a part of normal aging than as a critical indicator of depression. ${ }^{9}$ It can inaccurately be perceived as an understandable reaction to limitations imposed by medical conditions; retirement; death of a partner; decreasing social contacts; and lifestyle changes, such as moving to a care facility. ${ }^{10}$

Further, the additional MDD symptoms of changes in appetite, sleep disturbance, psychomotor agitation or retardation, fatigue, inappropriate guilt or feelings of worthlessness, poor concentration or indecisiveness, and recurrent thoughts of death or suicidal ideation can be overlooked in LDD. Loss of appetite and weight loss, a common and important symptom of LLD, is also associated with cognitive deficits ${ }^{21}$ and can predict an increased likelihood of developing dementia. ${ }^{22}$ Sleep disturbances, particularly early morning awakenings, are present in most cases of LLD and are predictive of a poor prognosis and future depressive episodes. ${ }^{3,23}$

Psychomotor retardation more so than psychomotor agitation is common in LLD. ${ }^{5}$ Although some slowing is not unexpected in normal aging, psychomotor retardation, such as disturbances in speech, facial expression, fine motor behavior, and gross locomotor activity (especially a decreased walking speed) are more apparent in people with LLD. ${ }^{24}$ Fatigue is often experienced as a physical tiring and lack of energy rather than as a mental fatigue. ${ }^{25}$ It is important to note that symptoms of frailty (including loss of interest, weight loss, psychomotor slowing, and physical tiring) overlap with symptoms of LLD and are frequently associated with comorbid medical concerns. $^{25}$

Inappropriate guilt or feelings of worthlessness are less frequent in older adults than in young people. ${ }^{20,26}$ By contrast, there is often actual impairment in peoples' ability to concentrate and make decisions. ${ }^{20}$ Further, compounding any assessment of how poor concentration and indecisiveness might be linked to depression is the question of whether medical conditions are causing disturbances in thinking and slowing information processing abilities. ${ }^{6}$ In LLD, the depressive symptom of "poor concentration" could be manifested more as memory loss or cognitive impairment with executive dysfunction (a clinical expression of frontostriatal abnormalities where the brain is less able to control cognitive processes, such as carrying out tasks). ${ }^{27}$ The cognitive impairments in LLD mimic symptoms of dementia. ${ }^{27}$

This cognitive impairment also makes people with LLD at high risk of death by suicide. ${ }^{28}$ Symptoms of recurrent thoughts of death or suicidal ideation in LLD are especially concerning. Older adults can act on their suicidal ideation with more serious intent, less warning, and more lethality than younger populations. ${ }^{29}$ Signs that indicate impending suicide include feelings of hopelessness or lack of purpose, feeling trapped, talking about death, preparing for suicide, social withdrawal, increased substance use, and reckless behavior. ${ }^{6}$ Factors believed to provide some protection against acting on suicidal thoughts include social connectedness, spirituality, religious beliefs, and cultural attitudes against suicide. ${ }^{6}$

For older adults, particularly those living with physical illnesses, increasing somatic complaints more so than typical symptoms of MDD are highly suggestive of LLD. ${ }^{30,31}$ Persistent complaints of pain, headache, fatigue, insomnia, gastrointestinal distress; unexplained delayed recoveries after surgery; and failure to thrive in institutional settings can be linked to depression. ${ }^{31}$ For people with cerebrovascular disease, any microdamage occurring in the small vessels compromises the ability of the frontal-subcortical circuits to regulate mood, resulting in a depression known as vascular or subcortical ischemic depression. ${ }^{32,33}$ Higher rates of LLD occur in people living with heart disease, diabetes, asthma, and arthritis. ${ }^{12}$ While it is understandable that people will experience distress caused by their physical illnesses, it is important to emphasize that unexplained somatic complaints are indicative of LLD.

Finally, people experiencing loneliness, or feeling as though they do not belong, are susceptible to LLD. ${ }^{34-36}$ Studies indicate that between $5 \%$ and $10 \%$ of people between the ages of 60 and 80 ; and between $40 \%$ and $50 \%$ of people over 80 reported feeling lonely frequently. ${ }^{37}$ Those at greatest risk of experiencing loneliness are women, particularly those who are older; and people who are without partners, are financially insecure and are physically unwell. ${ }^{37}$ As many as $87 \%$ of older adults with depressive symptoms reported cooccurring feelings of loneliness. ${ }^{36}$ Loneliness has been found to exacerbate depressive symptoms, decrease quality of life, and leave older adults feeling as though they have no control over their situation. ${ }^{36}$

In his seminal commentary on depression in later life, Dan Blazer urged clinicians not to limit their assessment of LLD to diagnostic categories of depression, but rather to include depressive symptoms that do not fully meet the criteria but nonetheless cause significant distress. ${ }^{20}$ As the preceding discussion explained, older adults with symptoms of depression without sadness, lack of vigor, apathy and withdrawal, loss of interest, loss of appetite, early morning awakenings, 
decreased walking speed, physical tiring, frailty, memory loss or cognitive impairment with executive dysfunction, suicidal ideation, somatic complaints, and loneliness could be suffering from LDD. Recognizing and addressing these symptoms can decrease depression and functional impairment and enhance quality of life for older adults. ${ }^{38}$ Next, actions that nurses can implement when they encounter the many older adults who are struggling with depression are suggested.

\section{3 | PRACTICE IMPLICATIONS: ACTIONS THAT CAN HELP}

\section{1 | Document symptoms of LLD}

Recognizing and documenting the aforementioned symptoms of LLD is a critical first step in helping people receive treatment for depression. Knowing that depressed older adults are more likely to present with loss of interest and unexplained somatic complaints than with a sad mood or self-identified complaints of depression, clinicians must intentionally inquire about LLD and maintain ongoing documentation. Seeking out information about any changes in lifestyle, social withdrawal, and feelings of loneliness from family members should also be included. Typical open ended interview questions that assess history, mental status, and level of cognitive and functional capacities should be followed by more directed questions specific to LLD. ${ }^{6}$

\section{2 | Screen with the Patient Health Questionaire-9}

Given the prevalence, impact and difficulties inherent in recognizing LLD, screening instruments can supplement interview findings and provide the objective data needed to indicate the risk or presence of depression in older adults. ${ }^{39}$ The 9-item Patient Health Questionnaire (PHQ-9), developed by Kroenke et al. ${ }^{40}$ provides reliable, valid detection of depression in LLD. ${ }^{6,10,38,39}$ The PHQ-9 is self-administered, requires about 5 minutes to complete, is easily scored and interpreted, and is available for free. ${ }^{40}$ The short questionnaire incorporates DSM-5 depression diagnostic criteria, as well as clinically significant depressive symptoms; rates the frequency of the symptoms and factors these into the scoring severity index; screens for suicidal ideation; and assigns weight to the depressive symptoms that impact how people function. ${ }^{40}$ The PHQ-9 can be administered in a variety of settings both as an initial screening instrument and to follow up and evaluate interventions. ${ }^{10,38}$

\section{3 | Assess suicide risk}

As with any depressive illness, suicidal ideation means that protective factors must be assessed in people who may be experiencing LLD. Suicidal ideation can range from thinking passively about death and wishing to die, to active thoughts of self-harm, plans, and an intention to die. ${ }^{6}$ In older adults, means can include but are not limited to, hanging, firearms, poisoning, and hoarding medications. ${ }^{6}$ Posing the question: "What stops you?" opens the door for people to express protective factors that are unique to their situation. Clearly, ongoing assessment, documentation, and immediate reporting of suicidal ideation must be implemented when LLD is or could be present.

\subsection{Pharmacological treatment}

Selective serotonin reuptake inhibitors (SSRIs) are the most common first-line treatment for LLD. ${ }^{1,41}$ The SSRI-Sertraline (Zoloft), has a favorable side-effect profile and can be taken by people with a history of cognitive impairment and stroke. ${ }^{41}$ When SSRIs are contraindicated, alternative classes of antidepressants, such as Mirtazapine (Remeron), demonstrate good efficacy in older adults. ${ }^{41}$ The side effects of Mirtazapine, which include sedation and weight gain, can have therapeutic benefit to the difficulty in sleeping and loss of appetite so commonly experienced in LLD. Tricyclic antidepressants, in particular, Nortriptyline (Allegron, Aventyl), can also be effective in older adults who do not have cardiac conditions or cognitive impairment. ${ }^{41}$

Antidepressant medications are usually trialed for approximately 6 weeks; should not be withdrawn abruptly and can be expected to be taken for at least 2 years. ${ }^{41}$ However, it is important to note that as many as one-third of older adults will not respond to antidepressant treatment; relapse rates for those who do respond are high; and the approach is less efficacious than for younger people. $^{13,42}$

\section{5 | Psychological treatment}

Psychological treatments, particularly when implemented in combination with pharmacological treatments have demonstrated moderate efficacy for some older adults. ${ }^{31,42,43}$ Cognitive behavioral therapy (CBT), problem-solving therapy (PST), interpersonal therapy (IPT), and reminiscence and life review therapy (RLRT) have been successfully implemented. ${ }^{43,44}$ In CBT, people identify and challenge distorted thinking; in PST, people identify problems, brainstorm solutions, implement a solution, and then evaluate its effectiveness; in IPT, people explore feelings and ways to change behavior in relation to interpersonal relationships, including grief and role transitions; and in RLRT people review positive life events to enhance wellbeing. ${ }^{43}$

Although therapies such as CBT, PST, and IPT are implemented by trained therapists, adaptions of RLRT and approaches to celebrating positive life events, both past and present, can readily be adapted in settings where nurses provide support to older adults. Similarly, behavioral activation (BA) therapy, which seeks to create a personal environment of positive reinforcement by increasing functional and pleasurable behavior, and by decreasing avoidant and depressed behavior, uses less complex protocols, and has successfully been implemented by mental health nurses. ${ }^{45}$ Many older adults, especially those who are over 75 and are experiencing depression for the first time, prefer not to talk about their depression and do not view pharmacological and psychological treatments as 
likely to be helpful. ${ }^{11}$ Therefore, exploring people's own ideas about the depression they are experiencing and the solutions they believe are valuable is foundational to any treatment approach.

\subsection{Electroconvulsive therapy}

Electroconvulsive therapy (ECT) can also be effective in treating LLD. ${ }^{7}$ People who will benefit most from ECT are those with depression that is resistant to other treatments and for those at risk of serious harm because of psychotic depression, suicidal ideation, or severe malnutrition. ${ }^{46}$ Although cardiovascular and cognitive side effects can occur with older adults, deleterious effects of ECT in LLD are usually limited and transient, with better cognitive outcomes with unilateral ECT. ${ }^{47}$

In one study, when elderly people with unipolar depression were treated twice weekly with right unilateral ECT, their cognition, psychomotor agitation, and psychomotor retardation improved significantly. ${ }^{48}$ This improvement was attributed to a significant gray matter volume increase, which was most pronounced on the side receiving neurostimulation. ${ }^{48}$ Common side effects of ECT that nurses must monitor include headache that usually responds to analgesics, temporary confusion or memory impairment, and falls immediately after treatment sessions. ${ }^{46}$

\section{7 | Neuromodulation therapies}

Alternative neuromodulation therapies also provide neurostimulation, but unlike ECT, these therapies do not require anesthetic and have reduced side effects, making them safer for older adults with comorbid medical conditions. ${ }^{49}$ Repetitive transcranial magnetic stimulation (rTMS) and transcranial direct-current stimulation (tDCS) modulate cerebral activity using brain stimulation and are usually implemented over a 4 to 6 week period. ${ }^{50}$ In older adults with nonpsychotic depression, rTMS is well tolerated but higher intensities and greater numbers of treatments may be needed than for younger people. ${ }^{50}$ Beyond its positive effects on mood, tDCS may also have positive effects on cognition. ${ }^{50}$

\section{8 $\quad$ Physical exercise}

Physical exercise programs can improve outcomes with some symptoms of LLD. In combination with antidepressant medication, exercise programs for older adults that included brief intervals of increased workload were found to decrease symptoms of depressed mood and psychomotor retardation. ${ }^{51}$ Aerobic activities, which stimulate and sustain heart and breathing rates, improved cognition and reduced disability in depressed older adults. ${ }^{52}$

When high intensity progressive resistance training was integrated into exercise programs, participants' sleep quality improved. ${ }^{53}$ Knowing that sleep disturbances are frequently present in LLD, ${ }^{3,23}$ the value of integrating resistance training into older adults' physical activities becomes clear. Weekly group exercise programs of $3 \times 50$ minute sessions, which included moderate cardiovascular exercise, such as walking with waving or clapping hands to music for a duration of 30 minutes, improved quality of life and perceived social support. ${ }^{54}$ Exergames or entertaining video games that combined game play with exercise decreased subsyndromal symptoms of depression ${ }^{55,56}$ and improved balance and mobility. ${ }^{55}$

In community settings, nurses can encourage older adults to participate in seniors' fitness activities in their area. In institutional settings, they can advocate for the creation of fitness programs that personalize physical exercise for patients/residents. Although medical conditions can impose limitations on peoples' mobility, engaging in some form of daily physical exercise can make an important difference in mood.

\section{9 | Lifestyle modifications}

In addition to increasing physical exercise, lifestyle modifications, such as improving dietary patterns and addressing vitamin D deficiencies may also exert a positive influence on some symptoms of depression. Emerging evidence suggests that modifying dietary patterns may reduce the risk of developing depression. ${ }^{57}$ Actions that can help include: following the Mediterranean diet; increasing consumption of fruits, vegetables, legumes, wholegrain cereals, nuts, and seeds; including a high consumption of foods rich in omega- 3 polyunsaturated fatty acids; replacing unhealthy foods with wholesome nutritious foods; and limiting intake of processed foods, "fast" foods, commercial bakery goods, and sweets. ${ }^{57}$

Although causal links between vitamin D deficiency and the development of depression have not been established, studies reveal that vitamin D deficiency accompanies late-life depression. ${ }^{58}$ For older adults, the skin capacity to synthesize vitamin $D$ from sunlight can be reduced as much as $25 \%$, increasing the likelihood of vitamin D deficiency. ${ }^{58}$ Those who have limited opportunities to be outdoors or to be exposed to sunlight are particularly at risk. The 25-hydroxy vitamin $\mathrm{D}$ blood test indicates that vitamin D deficiency exists when levels are less than $12.5 \mathrm{ng} / \mathrm{mL}^{59}$ Vitamin D supplements may be helpful.

\section{4 | CONCLUSION}

Adults over 60 can experience symptoms of depression somewhat differently than younger people. Older adults living with LLD may not appear sad or express feelings of depression. Instead, they may demonstrate loss of interest, frailty, cognitive impairment, suicidal ideation, unexplained somatic complaints, and loneliness. In response, nurses should document these symptoms, screen with the PAQ-9 and, importantly, assess for suicidal ideation. Antidepressant medications, $\mathrm{CBT}$, electroconvulsive therapy, neuromodulation therapies, physical exercise, and lifestyle modifications can bring needed support and relief to the increasing number of people burdened with LLD.

\section{CONFLICTS OF INTEREST}

The authors declare that there are no conflicts of interest. 


\section{ORCID}

Sherri Melrose (D) http://orcid.org/0000-0002-2056-8547

\section{REFERENCES}

1. Taylor WD. Depression in the elderly. $N$ Engl J Med. 2014;371(13):1228-1236. https://doi.org/10.1056/NEJMcp1402180

2. Djernes JK. Prevalence and predictors of depression in populations of elderly: a review. Acta Psychiatr Scand. 2006;113(5):372-387. https:// doi.org/10.1111/j.1600-0447.2006.00770.x

3. Ismail Z, Fischer C, McCall WV. What characterizes late-life depression? Psychiatr Clin North Am. 2013;36(4):483-496. https:// doi.org/10.1016/j.psc.2013.08.010

4. Luppa M, Sikorski C, Luck T, et al. Review: age- and gender-specific prevalence of depression in latest-life-systematic review and metaanalysis. J Affect Disord. 2012;136:212-221. https://doi.org/10.1016/j. jad.2010.11.033

5. Aziz R, Steffens DC. What are the causes of late-life depression? Psychiatr Clin North Am. 2013;36(4):497-516. https://doi.org/10. 1016/j.psc.2013.08.001

6. Glover J, Srinivasa S. Assessment and treatment of late-life depression. J Clin Outcomes Manag. 2017;24(3). https://www. mdedge.com/jcomjournal/article/145962/mental-health/assessmentand-treatment-late-life-depression

7. Kok RM, Reynolds CF. Management of depression in older adults a review. J Am Med Assoc. 2017;317(20):2114-2122. https://doi.org/10. 1001/jama.2017.5706

8. Lin JH, Huang MW, Wang DW, et al. Late-life depression and quality of life in a geriatric evaluation and management unit: an exploratory study. BMC Geriatr. 2014;14:77. http://www.biomedcentral.com/ 1471-2318/14/77

9. Padayachey U, Ramlall S, Chipps J. Depression in older adults: prevalence and risk factors in a primary health care sample. S Afr Fam Pract. 2017;59(2):61-66. https://doi.org/10.1080/20786190.2016. 1272250

10. Smith M, Haedtke C, Shibley D. Late life depression detection: an evidence-based guideline. J Gerontol Nurs. 2015;41(2):18-25. https:// doi.org/10.3928/00989134-20150115-99

11. Stark A, Kaduszkiewicz H, Stein J, et al. A qualitative study on older primary care patients' perspectives on depression and its treatments-potential barriers to and opportunities for managing depression. BMC Fam Pract. 2018;19(2):1-10. (2018). https://doi.org/10. 1186/s12875-017-0684-3

12. Yasamy M, Dua T, Harper M, Saxena S. Mental health of older adults, addressing a growing concern. Geneva: World Health Organization; 2013. http://www.who.int/mental_health/world-mental-health-day/ WHO_paper_wmhd_2013.pdf

13. Yoon S, Moon SS, Pitner R. Effective treatments of late-life depression in long-term care facilities: a systematic review. Res Soc Work Pract. 2018;28(2):116-130. https://doi.org/10.1177/1049731515621165

14. Espinoza R, Kaufman A. Diagnosis and treatment of late-life depression. Psychiatric Times. 2014;31(10). http://www.psychiatrictimes.com/ special-reports/diagnosis-and-treatment-late-life-depression

15. Pocklington C. Depression in older adults. Br J Med Pract. 2017;10(1): a1007.

16. American Psychiatric Association. Diagnostic and Statistical Manual of Mental Disorders DSM-5. 5th ed. Arlington, VA: American Psychiatric Publishing; 2013.

17. Covinsky KE, Cenzer IS, Yaffe K, O'Brien S, Blazer DG. Dysphoria and anhedonia as risk factors for disability or death in older persons: implications for the assessment of geriatric depression. Am J Geriatr Psychiatry. 2013;22(6):606-613. https://doi.org/10.1016/j.jagp.2012. 12.001
18. Gum AM, McDougal SJ, Mcllvane JM, Mingo CA. Older adults are less likely to identify depression without sadness. J Appl Gerontol. 2009;29(5):603-621. https://doi.org/10.1177/0733464809343106

19. Sachdev PS, Mohan A, Taylor L, Jeste DV. DSM-5 and mental disorders in older individuals: an overview. Harv Rev Psychiatry. 2015;23(5):320328. https://doi.org/10.1097/HRP.0000000000000090

20. Blazer DG. Depression in late life: review and commentary. J Gerontol. 2003;58a(3):249-265. https://doi.org/10.1093/gerona/ 58.3.M249

21. Potter GG, McQuoid DR, Steffens DC. Appetite loss and neurocognitive deficits in late-life depression. Int J Geriatr Psychiatry. 2015;30(6):647-654. https://doi.org/10.1002/gps.4196

22. Saha S, Hatch DJ, Hayden KM, Steffens DC, Potter GG. Appetite and weight loss symptoms in late life depression predict dementia outcomes. Am J Geriatr Psychiatry. 2016;24(10):870-878. https://doi. org/10.1016/j.jagp.2016.05.004

23. Kay DB, Dzierzewski JM. Sleep in the context of healthy aging and psychiatric syndromes. Sleep Med Clin. 2015;10(1):11-15. https://doi. org/10.1016/j.jsmc.2014.11.012

24. Bennabi D, Vandel P, Papaxanthis C, Pozzo T, Haffen E. Psychomotor retardation in depression: a systematic review of diagnostic, pathophysiologic, and therapeutic implications. Biomed Res Int. 2013;2013:18. Article ID 158746. https://doi.org/10.1155/2013/158746

25. Brown PJ, Rutherford BR, Yaffe K, et al. The depressed frail phenotype: the clinical manifestation of increased biological aging. Am J Geriatr Psychiatry. 2016;24(11):1084-1094. https://doi.org/10. 1016/j.jagp.2016.06.005

26. Haigh E (2016). Depression in community dwelling older adults: a broad brush stroke. Paper presented at the 26th Annual Maine Geriatrics Conference; June 3, 2016; Bar Harbor, ME.

27. Liguori C, Pierantozzi $M$, Chiaravalloti $A$, et al. When cognitive decline and depression coexist in the elderly: CSF biomarkers analysis can differentiate Alzheimer's disease from late-life depression. Front Aging Neurosci. 2018;10:38. https://doi.org/10.3389/fnagi. 2018.00038

28. Szanto K, Galfalvy H, Vanyukov PM, Keilp JG, Dombrovski AY. Pathways to late-life suicidal behavior: cluster analysis and predictive validation of suicidal behavior in a sample of older adults with major depression. J Clin Psychiatry. 2018;79(2):17m11611.

29. Ajilore O, Kumar A. Chapter 17: Suicide in late life. In: Dwivedi Y, ed. The Neurobiological Basis of Suicide. Boca Raton, FL: CRC Press/Taylor \& Francis; 2012. https://www.ncbi.nlm.nih.gov/books/NBK107214/

30. Hegeman JM, Kok RM, Van der Mast RC, Giltay EJ. Phenomenology of depression in older compared with younger adults: meta-analysis. Br J Psychiatry. 2012;200(4):275-281. https://doi.org/10.1192/bjp.bp. 111.095950

31. Blackburn P, Wilkins-Ho M, Wiese B. Depression in older adults: diagnosis and management. BC Med J. 2017;59(3):171-177.

32. Valkanova V, Ebmeier KP. Vascular risk factors and depression in later life: a systematic review and meta-analysis. Biol Psychiatry. 2013;73:406-413. https://doi.org/10.1016/j.biopsych.2012.10.028

33. Van Agtmaal MJM, Houben AJHM, Pouwer F, Stehouwer CDA, Schram MT. Association of microvascular dysfunction with late-life depression: a systematic review and meta-analysis. JAMA Psychiatry. 2017;74(7):729739. https://doi.org/10.1001/jamapsychiatry.2017.0984

34. Hegeman A, Schutter N, Comijs $\mathrm{H}$, et al. Loneliness and cardiovascular disease and the role of late-life depression. Int J Geriatr Psychiatry. 2018;33(1):e65-e72. https://doi.org/10.1002/gps.4716

35. Holvast F, Burger H, de Waal MMW, van Marwijk HWJ, Comijs HC, Verhaak PFM. Research report: Loneliness is associated with poor prognosis in late-life depression: longitudinal analysis of the Netherlands study of depression in older persons. J Affect Disord. 2015;185:1851-1857. https://doi.org/10.1016/j.jad.2015.06.036

36. Van Beljouw IMJ, Van Exel E, De Jong Gierveld J, et al. "Being all alone makes me sad": loneliness in older adults with depressive 
symptoms. Int Psychogeriatr. 2014;26(9):1541-1551. https://doi.org/ 10.1017/S1041610214000581

37. Hansen T, Slagsvold B. Late-life loneliness in 11 European countries: results from the generations and gender survey. Soc Indic Res. 2015;124(1):1-20.

38. Smith M, Stolder ME, Liu MF. Evaluation of an innovative late-life depression training program. Perspect Psychiatr Care. 2014;50:19-26. https://doi.org/10.1111/ppc.12019

39. Jones M. Using screening tools to identify the risk or presence of depression in older people. Nurs Times. 2009;105:49-50. https:// www.nursingtimes.net/roles/older-people-nurses/using-screeningtools-to-identify-the-risk-or-presence-of-depression-in-olderpeople-/5009615.article

40. Kroenke K, Spitzer RL, Williams JBW. The PHQ-9: validity of a brief depression severity measure. J Gen Intern Med. 2001;16(9):606-613. https://www.ncbi.nlm.nih.gov/pmc/articles/PMC1495268/

41. Allan CL, Ebmeier KP. Review of treatment for late-life depression. Adv Psychiatr Treat. 2013;19:302-309. https://doi.org/10.1192/apt. bp.112.010835

42. Knochel C, Alves G, Friedrichs B, et al. Treatment-resistant latelife depression: challenges and perspectives. Curr Neuropharmacol. 2015;13(5):577-591. https://doi.org/10.2174/1570159X130 5151013200032

43. Francis JL, Kumar A. Psychological treatment of late-life depression. Psychiatr Clin North Am. 2013;36:561-575.

44. Holvast F, Massoudi B, Oude Voshaar RC, Verhaak PFM. Nonpharmacological treatment for depressed older patients in primary care: a systematic review and meta-analysis. PLOS One. 2017;12(9): e0184666. https://doi.org/10.1371/journal.pone.0184666

45. Janssen $\mathrm{N}$, Huibers $\mathrm{MJH}$, Lucassen $\mathrm{P}$, et al. Behavioural activation by mental health nurses for late-life depression in primary care: a randomized controlled trial. BMC Psychiatry. 2017;17:230. https://doi. org/10.1186/s12888-017-1388-x

46. Unützer J. Late-life depression. N Engl J Med. 2007;357(22):2269-2276.

47. Kumar S, Mulsant B, Liu A, et al. Systematic review of cognitive effects of electroconvulsive therapy in late-life depression. Am J Geriatr Psychiatry. 2016;24(7):547-565. https://doi.org/10.1016/j.jagp.2016.02.053

48. Bouckaert F, De Winter FL, Emsell L, et al. Grey matter volume increase following electroconvulsive therapy in patients with late life depression: a longitudinal MRI study. J Psychiatry Neurosci. 2016;2):105-114. https://doi.org/10.1503/jpn.140322

49. McDonald WM. Neuromodulation treatments for geriatric mood and cognitive disorders. Am J Geriatr Psychiatry. 2016;24(12):1130-1141. https://doi.org/10.1016/j.jagp.2016.08.014
50. Gálvez V, Ho KA, Alonzo A, Martin D, George D, Loo CK. Neuromodulation therapies for geriatric depression. Curr Psychiatry Rep. 2015;17(7):59. https://doi.org/10.1007/s11920-015-0592-y

51. Murri MB, Ekkekakis $P$, Menchetti $M$, et al. Physical exercise for late-life depression: effects on symptom dimensions and time course. $J$ Affect Disord. 2018;230:65-70. https://doi.org/10.1016/j.jad.2018.01.004

52. Neviani F, Belvederi murri M, Mussi C, et al. Physical exercise for late life depression: effects on cognition and disability. Int Psychogeriatr. 2017;29(7):1105-1112. https://doi.org/10.1017/S1041610217000576

53. Singh NA, Stavrinos TM, Scarbek Y, Galambos G, Liber C, Fiatarone Singh MA. A randomized controlled trial of high versus low intensity weight training versus general practitioner care for clinical depression in older adults. J Gerontol A Biol Sci Med Sci. 2006;60(6):768-776.

54. Huang TT, Liu CB, Tsai YH, Chin YF, Wong CH. Physical fitness exercise versus cognitive behavior therapy on reducing the depressive symptoms among community-dwelling elderly adults: a randomized controlled trial. Int J Nurs Stud. 2015;52(10):1542-1552. https://doi.org/10.1016/j.ijnurstu.2015.05.013

55. Chao YY, Scherer YK, Montgomery CA, Wu YW, Lucke KT. Physical and psychosocial effects of Wii Fit exergames use in assisted living residents: a pilot study. Clin Nurs Res. 2015;24(6):589-603. https:// doi.org/10.1177/1054773814562880

56. Rosenberg D, Depp CA, Vahia IV, et al. Exergames for subsyndromal depression in older adults: a pilot study of a novel intervention. Am J Geriatr Psychiatry. 2010;18(3):221-226. https://doi.org/10.1097/JGP. Ob013e3181c534b5

57. Opie RS, Itsiopoulos C, Parletta N, et al. Dietary recommendations for the prevention of depression. Nutr Neurosci. 2017;20(3):161-171. https://doi.org/10.1179/1476830515Y.0000000043

58. Jovanova O, Aarts N, Noordam R, Carola-Zillikens M, Hofman A, Tiemeier $\mathrm{H}$. Vitamin $\mathrm{D}$ serum levels are cross-sectionally but not prospectively associated with late-life depression. Acta Psychiatr Scand. 2017;135:185-194. https://doi.org/10.1111/acps.12689

59. Manson JE, Brannon PM, Rosen CJ, Taylor CL. Vitamin D deficiencyis there really a pandemic? N Engl J Med. 2016;375:1817-1820. https://doi.org/10.1056/NEJMp1608005

How to cite this article: Melrose S. Late life depression: nursing actions that can help. Perspect Psychiatr Care. 2019;55: 453-458. https://doi.org/10.1111/ppc.12341 